EPJ Web of Conferences 116, 06009 (2016)

DOI: $10.1051 /$ epjconf/201611606009

(C) Owned by the authors, published by EDP Sciences, 2016

\title{
Measurement of light scattering in deep sea
}

\author{
N. Maragos, K. Balasi, T. Domvoglou, I. Kiskiras, D. Lenis, M. Maniatis, and G. Stavropoulos \\ NCSR DEMOKRITOS, Institute for Nuclear and Particle Physics, Agia Paraskevi 153 42, Greece
}

\begin{abstract}
The deep-sea neutrino telescope in the Mediterranean Sea, being prepared by the KM3NET collaboration, will contain thousands of optical sensors to readout. The accurate knowledge of the optical properties of deep-sea water is of great importance for the neutrino event reconstruction process. In this study we describe our progress in designing an experimental setup and studying a method to measure the parameters describing the absorption and scattering characteristics of deep-sea water. Three PMTs will be used to measure in situ the scattered light emitted from six laser diodes in three different wavelengths covering the Cherenkov radiation spectrum. The technique for the evaluation of the parameters is based on Monte Carlo simulations and our results show that we are able to determine these parameters with satisfying precision.
\end{abstract}

\section{Introduction}

The sensors of the deep-sea neutrino telescope KM3NET will detect the Cherenkov photons, emitted by the charged secondary particles created by neutrino interactions in the detector medium. The study presented below is motivated by the need of achieving an accurate description of the formation of the sensors signal, which the simulation and reconstruction techniques mainly rely on. This means that the characteristics of the absorption and scattering of the Cherenkov light, have to be determined as accurate as possible.

An experimental setup to measure in situ the optical properties of the detector medium is being studied and work concerning the design and the data analysis methods to be used, is presented below. In Sect. 2 a description of the experimental setup is made, including the specifications of the detector, the light sources and details about the geometry and the mechanical structure to be used. Section 3 is dedicated to the description of the Monte Carlo simulation background theory, its implementation details and the description of the method used for the parameters estimation.

\section{The experimental setup}

\subsection{Overview}

The measuring system will consist of light sources and light detectors. Photons emitted from the light sources will be scattered by sea water and detected by light detectors at fixed distances giving information about the optical properties of the medium. Sources and Detector will be placed inside of

This is an Open Access article distributed under the terms of the Creative Commons Attribution License 4.0, which permits unrestricted use, distribution, and reproduction in any medium, provided the original work is properly cited. 


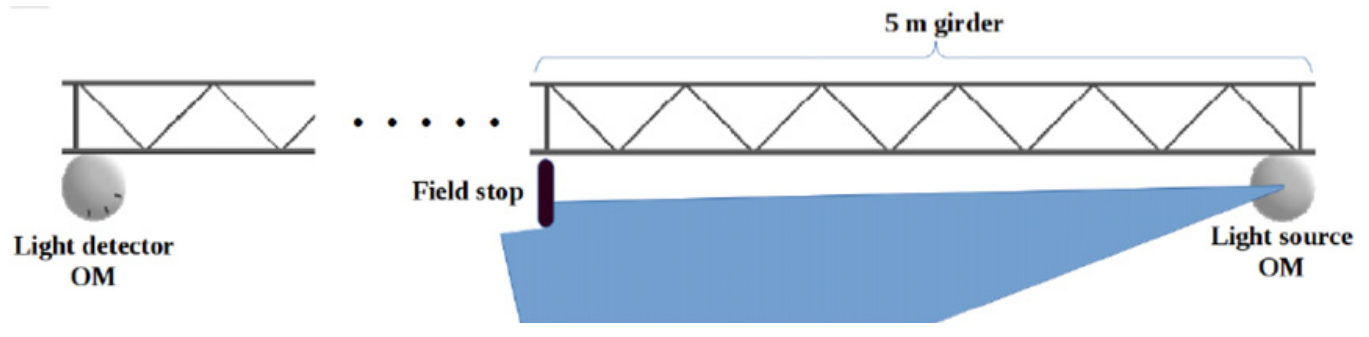

Figure 1. The experimental setup.
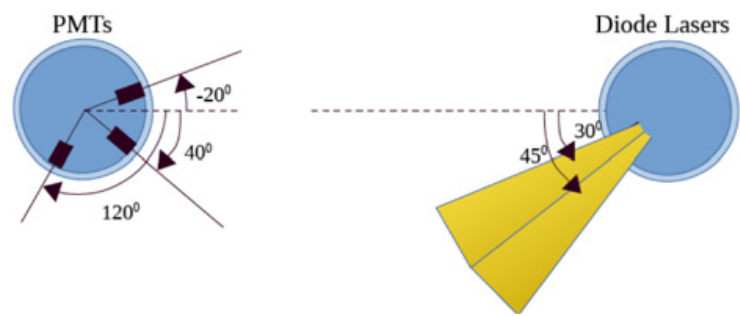

Figure 2. left: the detector Optical Module with three PMTs viewing at directions - 20, 40, 120 deg with respect to the axis joining the centers of the Optical Modules. Right: the light source Optical Module with one pare of lasers emitting in 30 and $45 \mathrm{deg}$.

pressure resistant $1.5 \mathrm{~cm}$ thick glass spheres of outer diameter $43.2 \mathrm{~cm}$ (optical modules, OMs). The mechanical frame supporting both spheres will consist of $5 \mathrm{~m}$ long titanium girders attached to each other to form a long linear structure. The light source OM will be attached at the outer side and in the front end of the first girder (Fig. 1). The detector OM will be attached opposite to the light source OM and in the rear end of the last girder. A metal circular disk will be placed in front of the light source $\mathrm{OM}$ at the rear end of the first girder, preventing unscattered light from being detected. Three different separation distances will be used inserting zero, one and two girders in-between the first and the last $(\sim 10,15$ and $20 \mathrm{~m})$.

\subsection{Light source OM}

The light source will consist of three pairs of laser diodes emitting in three different wavelengths $(405 \pm 2 \mathrm{~nm}, 450 \pm 2 \mathrm{~nm}, 520 \pm 2 \mathrm{~nm})$ covering the Cherenkov spectrum. The orientation of the two laser diodes in each pair will be $30^{\circ}$ and $45^{\circ}$ with respect to the axis formed by the centers of the OMs and in direction opposite to the mechanical frame (Fig. 2). The laser diodes will emit light pulses with pulse width $10 \mathrm{~ns}$, pulse energy $2 \mathrm{pJ}$ and repetition rate around $1 \mathrm{MHz}$. Their beam divergence will be $\sim 4$ degrees.

\subsection{Detector OM}

The detector will consist of three HAMAMATSU H10682-210 PMTs modules operating at photon counting mode with their field of view centered at directions $-20^{\circ}, 40^{\circ}$ and $120^{\circ}$ with respect to the axis formed by the centers of the OMs (Fig. 2). These PMTs will measure the detected photon arrival times inside $1 \mu \mathrm{s}$ "time windows" triggered together with the light pulses emitted from the laser diodes. The measured time accuracy will be $2.5 \mathrm{~ns}$. 


\subsection{Data acquisition}

The MAIN COUNTER works at $400 \mathrm{MHz}$, giving a time resolution of $2.5 \mathrm{~ns}$. Every $1280 \mathrm{~ns}$ the counter restarts and at the same time a signal is send to the light souces triggering a light pulse. Each time a PMT gives a hit signal a buffer holds the MAIN COUNTER. The number of emitted laser pulses are counted and there is a record every 1024 pulses along with a mask indicating the active laser. Just before the MAIN COUTER restarts the PMT registers are guided to a FIFO along with a mask indicating which PMT gave the signal. A micro-controller is responsible for choosing witch laser will be active and for how long. It is also responsible for transferring the data to an SD card.

\section{Simulation and parameter estimation technique}

\subsection{Theoretical model used for the simulations}

The scattering model used for this study is a combination of Mie and Rayleigh scattering [1]. Its scattering angle distribution is expressed as a weighted sum of molecular and particulate scattering:

$$
\frac{\mathrm{dP}}{\mathrm{d} \Omega_{\mathrm{s}}}=\mathrm{F}\left(\cos \theta_{\mathrm{s}} ; \mathrm{p}, \mathrm{a}_{\text {Rayl }}, \mathrm{a}_{\text {Mie }}\right)=\mathrm{p} \times \mathrm{g}\left(\mathrm{a}_{\text {Rayl }}, \cos \theta_{\mathrm{s}}\right)+(1-\mathrm{p}) \times \mathrm{f}\left(\mathrm{a}_{\text {Mie }}, \cos \theta_{\mathrm{s}}\right)
$$

where $\theta_{\mathrm{s}}$ is the scattering angle, $\mathrm{p}$ the Rayleigh contribution, $\mathrm{a}_{\mathrm{Mie}}$ is the average cosine of the scattering angle for Mie scattering and $\mathrm{a}_{\text {Rayl }}$ defines the shape of the Rayleigh scattering function. $\mathrm{f}\left(\mathrm{a}_{\mathrm{Mie}}, \cos \theta_{\mathrm{s}}\right)$ is a Henyey-Greenstein function given by:

$$
f\left(\mathrm{a}_{\mathrm{Mie}}, \cos \theta_{\mathrm{s}}\right)=\frac{1}{4 \pi} \frac{\left(1-\mathrm{a}_{\mathrm{Mie}}^{2}\right)}{\left(1+\mathrm{a}_{\mathrm{Mie}}^{2}-2 \mathrm{a}_{\mathrm{Mie}} \cos \theta_{\mathrm{s}}\right)^{\frac{3}{2}}} .
$$

The phase function $\mathrm{g}\left(\mathrm{a}_{\text {Rayl }}, \cos \theta_{\mathrm{s}}\right)$, which is describing the Rayleigh scattering from sea water molecules, has been parametrized as:

$$
\mathrm{g}\left(\mathrm{a}_{\text {Rayl }}, \cos \theta_{\mathrm{s}}\right)=\frac{\left(1+\mathrm{a}_{\text {Rayl }} \cos ^{2} \theta_{\mathrm{s}}\right)}{4 \pi\left(1+\frac{1}{3} \mathrm{a}_{\text {Rayl }}\right)} .
$$

\subsection{Method of parameter estimation}

The experimental setup is simulated with the use of GEANT4 packages. Monte Carlo photon events are generated with their propagation defined by the described theoretical model. In a real life experiment, the $3 \times 6$ arrival time distributions from the measured data would be compared with the corresponding Monde Carlo (MC) distributions (Fig. 3). In this study we produced an independent MC sample of events which is considered as the data set (hereafter pseudo-data).

Since in the real experiment we won't know the real values of the parameters, we conduct another MC simulation with parameters different from that of the pseudo-data and we try to fit the corresponding arrival time histograms to the pseudo-data. The technique we use for the $\chi^{2}$ fit relies on re-weighting the photon events according to their track probability [2]:

$$
\mathrm{P}\left(\mathrm{L}_{\mathrm{a}}, \mathrm{L}_{\mathrm{s}}, \mathrm{p}, \mathrm{a}_{\text {Rayl }}, \mathrm{a}_{\text {Mie }}\right) \propto \mathrm{e}^{-\mathrm{L} / \mathrm{L}_{\mathrm{a}}} \times\left[\prod_{\mathrm{i}=1}^{\mathrm{N}-1} \frac{1}{\mathrm{~L}_{\mathrm{s}}} \mathrm{F}\left(\cos \theta_{\mathrm{i}} ; \mathrm{p}, \mathrm{a}_{\text {Rayl }}, \mathrm{a}_{\text {Mie }}\right)\right] \times \mathrm{e}^{-\mathrm{l}_{\mathrm{N}} / \mathrm{L}_{\mathrm{s}}},
$$

where $L=\sum_{i=1}^{N} l_{i}$ is the total photon track length, $L_{a}$ is the absorption length and $L_{s}$ is the scattering length. The right part of (4) consists of three terms. $e^{-L / L_{a}}$ is the probability for a photon not to be absorbed after traveling length $L$, the middle term is proportional to the probability for a photon to be 

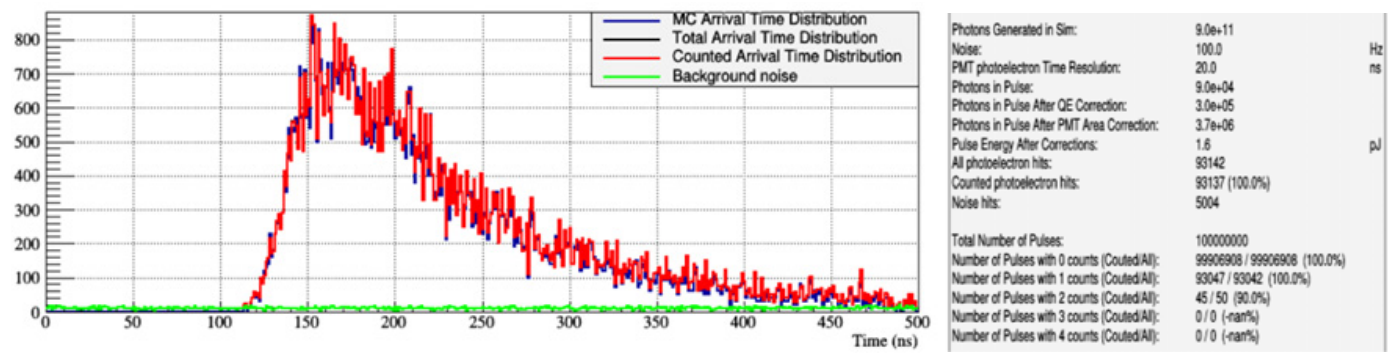

Figure 3. PMT hit arrival time distribution derived from simulation.

scattered with an angle $\theta_{i}$ after having crossed a distance $l_{i}$, and $e^{-l_{N} / L_{s}}$ gives the probability for the photon not to be scattered at the last segment.

Using the estimated parameters from the fit we produce an additional set of MC events and we make a new fit to estimate again the parameters. We repeat this process until simulation and estimated parameters converge and their difference does not exceed $1 \sigma$.

To test the reliability of the fit method we make an application of it using the following two sets of parameters for pseudo-data and simulation respectively:

$$
\begin{aligned}
& L_{a}=73 \mathrm{~m}, \quad L_{s}=56.4 \mathrm{~m}, \quad p=0.21, \quad a_{\text {Mie }}=0.75, \quad a_{\text {Rayl }}=0.853 \quad \text { pseudo-data } \\
& L_{a}=65 \mathrm{~m}, \quad L_{s}=48.4 \mathrm{~m}, \quad p=0.17, \quad a_{\text {Mie }}=0.924, \quad a_{\text {Rayl }}=0.853 \quad \text { simulation data. }
\end{aligned}
$$

The minimization process is made in the five dimensional parameter space. We set $a_{\text {Rayl }}=0.853$ fixed, since it is a well known factor, attributable to the anisotropy of water molecules [3], and additionally its variations have insignificant impact in the resulting arrival time distributions. We also insert a normalization parameter $\mathrm{c}$ that can be related with the total number of generated photons. Using the ROOT MINUIT package we get the parameter estimations and the correlation matrices. A convergence is reached after five repeats of the fit process. The "real" parameters are well estimated from the final fit with the following uncertainties:

$$
\delta \mathrm{p}=0.0019(0.9 \%), \quad \delta L_{s}=1.34 \mathrm{~m}(2.4 \%), \quad \delta \mathrm{L}_{\mathrm{a}}=2.14 \mathrm{~m}(2.9 \%) \quad \text { and } \delta \mathrm{a}_{\mathrm{Mie}}=0.0023(0.3 \%) .
$$

\section{Conclusions}

Motivated by the need of accurate knowledge of the parameters determining the behavior of Cherenkov light in the deep-sea water medium for the KM3NeT we studied the design of an experimental setup using diode lasers and PMT sensors inside pressure resistant Optical Modules dedicated for in situ measurements. We investigated important parameters of the setup and we studied and tested a method to estimate the absorption and scattering parameters by fitting model based re-weighted Monte Carlo simulations to the expected PMT photon arrival time distributions. Our test results show that the accuracy of the parameter estimation is $\sim 2 \%$ for absorption and scattering lengths and less than $1 \%$ for the Rayleigh contribution and Mie angular distribution factor. Guided from the present design study we plan to realize the experiment.

Financial support through the European Fund for Regional Development, NSRF 2007-2013 action "Development of Research Centers - КРНПІ $\Sigma$ " is acknowledged. 
Very Large Volume Neutrino Telescope (VLVnT-2015)

\section{References}

[1] D.J.L. Bailey, Wolfson College, Oxford, thesis (2002)

[2] A. Papaoikonomou, A Leisos, A. Tsirigotis and S. Tzamarias, Workshop on very large volume neutrino telescopes, Stockholm, Sweden (2013)

[3] J.A. Aguilar et al., Astroparticle Physics, v23 (2005) 